\title{
The management of macular hole retinal detachment and macular retinoschisis in pathological myopia; a UK collaborative study
}

\author{
Heidi Laviers ${ }^{1}$ - Ji-Peng Olivia Li ${ }^{2}$ - Anna Grabowska ${ }^{2}$ Stephen J. Charles ${ }^{3}$ - David Charteris ${ }^{2} \cdot$ Richard J. Haynes $^{4}$. \\ D. Alistair H. Laidlaw ${ }^{5}$ - David H. Steel ${ }^{6,7}$ - David Yorston ${ }^{8}$ • Tom H. Williamson ${ }^{5}$ - Hadi Zambarakji ${ }^{1}$. \\ On behalf of the COllaboration of British RetinAl Surgeons (COBRA) study group
}

Received: 23 June 2017 / Revised: 6 June 2018 / Accepted: 11 June 2018 / Published online: 16 July 2018

(c) The Royal College of Ophthalmologists 2018

\begin{abstract}
Purpose To study UK practice patterns for the management of retinal detachment secondary to macular hole (MHRD) and macular retinoschisis (MRS) in pathological myopia (PM). To review the anatomical and visual outcomes of the surgically managed cases.

Methods A prospective observational case series for the management of MHRD was undertaken in association with the British Ophthalmological Surveillance Unit (BOSU). The results were combined with retrospective data, collected by the COllaboration of British RetinAl Surgeons (COBRA), on the management of both MHRD and MRS in PM in the UK. A total of 20 cases of MHRD and 53 cases of MRS (27 surgical cases and 26 cases managed conservatively) are reported in this combined study.

Results MHRD: Mean baseline best corrected visual acuity (BCVA) was $1.60 \log$ MAR. All cases underwent pars plana vitrectomy (PPV). Mean post-operative BCVA was $1.49 \operatorname{logMAR}(p=0.674)$. The macular hole was closed in $5 / 20(25 \%)$ cases, open/flat in 10/20 (50\%) cases and open/elevated in 4/20 cases (20\%). MRS: Mean baseline BCVA was 0.87 logMAR in the surgical group and $0.45 \log$ MAR in the conservatively managed group $(p=0.002)$. All eyes that had surgical intervention underwent PPV. Mean post-operative BCVA was $0.68 \log$ MAR $(p=0.183)$. Anatomical outcomes demonstrated a persistent MRS in 2/27 (7.4\%) cases, partial resolution in 7/27 (25.9\%) cases and complete resolution in 16/27 $(59.2 \%)$ cases.

Conclusions PPV is the only surgical procedure performed for the management of MHRD and MRS amongst the study participants. Success rates and visual outcomes are limited for MHRD and consistent with the current literature for MRS.
\end{abstract}

Members of the COllaboration of British RetinAl Surgeons (COBRA) study group are listed below Acknowledgements.

Electronic supplementary material The online version of this article (https://doi.org/10.1038/s41433-018-0166-4) contains supplementary material, which is available to authorized users.

Hadi Zambarakji

zambarakji@doctors.org.uk

1 The Eye Treatment Centre, Whipps Cross University Hospital, Barts Health NHS Foundation Trust, London, UK

2 Moorfields Eye Hospital NHS Foundation Trust, London, UK

3 Manchester Royal Eye Hospital, Central Manchester University Hospitals NHS Foundation Trust, Manchester, UK

4 Bristol Eye Hospital, University Hospitals Bristol NHS

\section{Introduction}

\section{Pathogenesis}

Pathological myopia (PM) is a common cause of vision loss worldwide and the global prevalence appears to be increasing, particularly in Asian populations $[1,2]$.

\section{Foundation Trust, Bristol, UK}

5 Ophthalmology Department, Guy's and St Thomas' NHS Foundation Trust, London, UK

6 Sunderland Eye Infirmary, NHS Trust, Sunderland, UK

7 Institute of Genetic Medicine, Newcastle University, Newcastle Upon Tyne, UK

8 Tennent Institute of Ophthalmology, Gartnavel Hospital, NHS Greater Glasgow and Clyde, Glasgow, UK 
Prevalence estimates have been difficult to ascertain due to varying study definitions and differences between populations. The estimated prevalence in the USA, Western Europe and Australia is 2.8-4.6\% [1, 2]. The definition of PM has been recently updated and is defined as eyes with characteristic myopic fundal findings and a refractive error of $\geq-8$ Dioptres [3, 4].

Amongst the spectrum of complications associated with PM, macular hole with secondary retinal detachment (MHRD) and macular retinoschisis (MRS) have been difficult to manage $[5,6]$.

While the exact pathogenesis of MHRD is unknown, many hypotheses have been suggested including anterior-posterior vitreoretinal tractional forces, posterior staphyloma and large areas of chorioretinal atrophy leading to weak adhesion between the retina and the underlying retinal pigment epithelium (RPE) [7-9].

In 1999, Akiba et al. were the first to report the role of staphyloma in contributing to retinal detachment in the presence of macular hole rather than anterio-posterior vitreomacular traction [10]. During a similar period, Takano et al. also found that retinal detachment and MRS were common features in high myopes with staphyloma, but postulated that retinal detachment may precede macular hole formation [11]. With increasing age, the incidence and size of the staphyloma increase. This may be associated with progressive stretching and thinning of the retina because of the disparity between the retina and the sclera, which could precipitate the development of retinal detachment or retinoschisis [12].

High resolution optical coherence topography (OCT) provides a detailed examination of the retinal structure and allows the distinction between macular detachment and retinoschisis [13]. MRS in PM is now considered a progressive condition leading to macular hole and secondary retinal detachment when myopic traction maculopathy is present, with high rates of progression in cases of extensive MRS [14-23]. It would, therefore, appear that both intraocular and outer ocular wall factors can impact on the natural progression to MHRD and the outcome of treatment strategies.

The treatment criteria and management of MHRD and MRS also remain controversial [6]. Pars plana vitrectomy (PPV), encircling scleral buckle, peeling of internal limiting membrane (ILM)/pre-retinal membranes, various intraocular tamponade agents and macular buckle have all been used with variable success rates. Traditionally, the management in the UK has involved PPV, epiretinal membrane (ERM)/ ILM peel with gas or oil tamponade [24]. While macular buckling for MHRD was originally developed to treat the posterior staphyloma, further interest in this technique has increased during the last 10-15 years, at least in part because of the limited success rates with vitrectomy alone [5, 25-28].
The aim of this study is to gain an understanding of the UK practice patterns for the management of MHRD and MRS in PM. The study also aims to review the anatomical and visual outcomes of the surgical management of these cases.

\section{Methods}

A prospective observational case series for the management of MHRD was carried out in association with the British Ophthalmological Surveillance Unit (BOSU). BOSU data were combined with data from a retrospective COllaboration of British RetinAl Surgeons (COBRA) data provided by a group of UK vitreoretinal surgeons. Retrospective COBRA data included cases of MHRD that had been managed surgically in addition to cases of MRS, which had either undergone surgical or conservative management.

Initial case ascertainment was through the BOSU reporting system from October 2013 to October 2014 in collaboration with the Royal College of Ophthalmologists and covered Britain and Northern Ireland. The retrospective COBRA data was based on consecutive cases collected by a group of nine British Vitreoretinal surgeons from April 2011 to April 2016. A standardised questionnaire had been designed and approved by the BOSU steering group after refinement by interested Ophthalmologists.

BOSU provides an established infrastructure to prospectively investigate ophthalmic conditions with important public health implications in the UK. All Consultant Ophthalmologists in the UK were sent a monthly report card to report any case of myopia associated MHRD during the surveillance period. The case definition was as noted in the methods section of the present paper. The BOSU questionnaire was sent to all reporting UK Ophthalmologists immediately on receiving notification from the BOSU team and a further follow-up questionnaire was sent 6 months later. The same initial and follow-up questionnaires were utilised in the retrospective COBRA study and sent to the study participants. Case definition and completeness of response was validated by the principle contact at the point of data entry; reporting ophthalmologists were contacted for further information as necessary. The questionnaire included details on the following variables and potential confounders: age, gender, lens status, axial length, macular pathology, surgical procedures, endotamponade utilised, posturing regime, baseline best corrected visual acuity (BCVA) and post-operative visual and anatomical outcomes in the cases that were surgically managed.

Visual acuity data were reported in either Snellen acuity or logMar units and where necessary converted to logMar equivalent. Data were analysed using Stata 14.1 (StataCorp LP) software. Analyses were performed with the Pearson $X^{2}$ 
test, Student's $t$-test and analysis of variance (ANOVA). A $p$-value of $<0.05$ was taken as statistically significant. Only those variables showing significant associations on univariate analysis were included in the multivariate models.

\section{Case definitions}

At the time of the current study, the definition of PM was widely accepted as a spherical equivalent refractive error of $\geq-6$ Dioptres or an axial length of $\geq 25.5 \mathrm{~mm}$ with characteristic degenerative changes [3]. While this has been updated more recently to eyes with characteristic myopic fundal findings and a refractive error of $\geq-8$ Dioptres, the former definition of PM has been used for both the BOSU data and the COBRA data [4].

We included all cases of MHRD, which was defined as the separation of the sensory retina from the RPE by subretinal fluid (SRF) extending at least as far as the edge of the temporal retinal vasculature around the macula where the macular hole is the primary break that resulted in retinal detachment. The presence of retinal detachment was confirmed using OCT. The post-operative anatomic outcome was based on the macular hole appearance using OCT at 6 months where the macular hole appearance is defined as open/flat, open/elevated or closed. Retinal reattachment based on OCT at 6 months is defined as attachment of the retina without any tamponade present and no SRF present, which could spread. This would include eyes with anterior fluid walled off by $360^{\circ}$ retinopexy.

We included all cases with MRS based on the OCT appearance, which was defined as splitting of the inner or outer plexiform layers of the retina. The post-operative anatomic outcome was based on the OCT appearance of the MRS at 6 months, based on the complete or partial resolution of the retinoschisis.

Patients with peripheral retinal breaks and a peripheral retinal detachment at presentation were excluded from the study.

The study obtained ethical approval from Whipps Cross University Hospital, Barts Health NHS Trust ethics committee and adhered to the tenets of the Declaration of Helsinki. The study used non-identifiable data collected during the course of routine clinical practice.

\section{Results}

\section{MHRD}

A total of 20 cases of MHRD were identified. Ten cases were recruited from the prospective study and 10 cases were recruited from the retrospective study. The mean age of participants at baseline was 63.9 years (range $37-83$ years)
Table 1 Baseline characteristics and surgical techniques used in the management of MHRD

\begin{tabular}{|c|c|}
\hline & Mean $(\mathrm{CI}) n=20$ \\
\hline Mean age & $63.9(59.1-68.7)$ \\
\hline Mean baseline BCVA (logMAR) & $1.60(1.21-1.98)$ \\
\hline Gender & $n(\%) n=20$ \\
\hline Male & $6(30.0)$ \\
\hline Female & $14(70.0)$ \\
\hline Axial length & $n(\%) n=19^{\mathrm{a}}$ \\
\hline $25.5-30.0 \mathrm{~mm}$ & $10(52.6)$ \\
\hline$>30.0 \mathrm{~mm}$ & $9(47.4)$ \\
\hline Lens status & $n(\%) n=18^{\mathrm{b}}$ \\
\hline Clear & $8(44.4)$ \\
\hline Cataract & $3(16.7)$ \\
\hline Posterior chamber IOL & $7(38.9)$ \\
\hline Macular pathology & $n(\%) n=19^{\mathrm{a}}$ \\
\hline Posterior staphyloma & $16(84.2)$ \\
\hline Non-foveal macular hole & $1(5.3)$ \\
\hline Post-vitreous detachment & $10(52.6)$ \\
\hline Macular retinoschisis & $9(47.4)$ \\
\hline Chorioretinal atrophy & $7(36.8)$ \\
\hline Epiretinal membrane & $6(31.6)$ \\
\hline Choroidal neovascularisation & $1(5.3)$ \\
\hline Surgical procedures & $n(\%) n=19^{\mathrm{a}}$ \\
\hline Post-vitreous detachment induced & $8(42.1)$ \\
\hline Epiretinal membrane peeled & $8(42.1)$ \\
\hline Internal limiting membrane peeled & $14(73.7)$ \\
\hline Stain & $13(68.4)$ \\
\hline Laser retinopexy & $0(0.0)$ \\
\hline Explant & $0(0.0)$ \\
\hline Endotamponade & $n(\%) n=20$ \\
\hline None & $0(0.0)$ \\
\hline Air & $0(0.0)$ \\
\hline SF6 & $1(5.0)$ \\
\hline $\mathrm{C} 2 \mathrm{~F} 6$ & $9(45.0)$ \\
\hline $\mathrm{C} 3 \mathrm{~F} 8$ & $7(35.0)$ \\
\hline Silicone/heavy oil & $3(15.0)$ \\
\hline Posturing & $n(\%) n=20$ \\
\hline None & $3(15.0)$ \\
\hline$<24 \mathrm{~h}$ & $0(0.0)$ \\
\hline 1-7 days & $11(55.0)$ \\
\hline$>7$ days & $6(30.0)$ \\
\hline
\end{tabular}

SF6 sulphur hexafluoride, C2F6 hexafluoroethane, $C 3 F 8$ perfluoropropane

${ }^{a}$ Data missing in one case

${ }^{\mathrm{b}}$ Two cases

and included 14 (70\%) female participants. Baseline characteristics included a mean pre-operatively BCVA of 1.60 $\log$ MAR (range: $0.2-3.0$ ) with an axial length of 25.5-30.0 
$\mathrm{mm}$ in $10 / 20$ cases and $>30.0 \mathrm{~mm}$ in $9 / 20$ cases (one case not specified). The duration of central visual loss was $<1$ week in three cases, 1-4 weeks in four cases and $>4$ weeks in 13 cases. Baseline macular status is highlighted in Table 1 (data were missing for one case) and demonstrates a high proportion with posterior staphyloma (16/19 cases) and MRS (9/19 cases).

All cases were managed by PPV with gas or oil tamponade as demonstrated in Table 1 . The most frequently stated endotamponade used was C2F6 in 9/20 (45\%) cases and drainage of the SRF through the macular hole was carried out in 16/20 (80\%) cases. Posturing regimes varied but the most frequently stated posturing regime was for 1-7 days in $11 / 20(55 \%)$ cases.

Mean follow-up was for a period of 6.3 months (range: 6-12 months) and BCVA remained stable with a mean of $1.60 \log$ MAR at baseline and a mean of $1.49 \log$ MAR (range: $0.4-3.0)$ post-operatively $(p=0.674)$. BCVA decreased post-operatively in $5 / 20(25 \%)$ cases, was unchanged in $3 / 20(15 \%)$ cases and improved in $12 / 20$ (60\%) cases. A total of $8 / 20(40 \%)$ cases improved by $>0.30 \log$ MAR units or 2 Snellen lines (Fig. 1). Older age was associated with improved BCVA on univariate analysis $(p=0.039)$ and the use of C2F6 had a borderline association with improved BCVA $(p=0.05)$. However, these variables were no longer significantly associated when carried through to multivariate analysis.

The macular hole was reported as closed in 5/20 (25\%) cases, open and flat in 10/20 (50\%) cases and open and elevated in 4/20 (20\%) cases (with one case undetermined due to difficulty in interpreting the OCT findings). The presence of MRS was significantly associated with an open/elevated MH $(p=0.024)$. A post-operatively open/flat MH demonstrated a mean BCVA of $1.76 \log$ MAR at baseline and a mean BCVA of $1.41 \log$ MAR post-operatively $(p=0.366)$. A postoperatively open/elevated MH demonstrated a mean BCVA

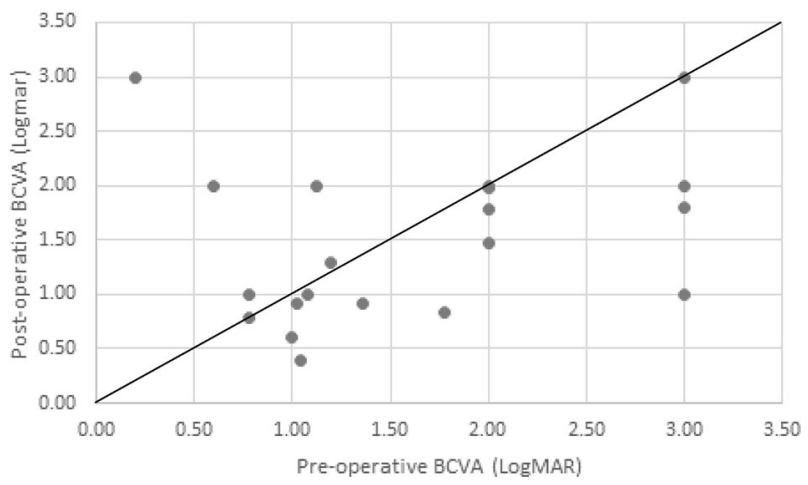

Fig. 1 A plot of post-operative and pre-operative BCVA (logMAR) in cases of MHRD. The line of equality represents no change in BCVA with the points below the line indicating an improvement in BCVA and those above the line indicating a reduction in post-operative BCVA of $1.29 \log$ MAR pre-operatively and a mean of $1.98 \mathrm{log}$ MAR post-operatively $(p=0.393)$. A post-operatively closed MH demonstrated a mean BCVA of $1.43 \log$ MAR pre-operatively and a mean of $1.26 \log$ MAR postoperatively $(p=0.651)$.

Post-operatively $4 / 20$ cases had SRF at 6 months giving a primary retinal reattachment rate of $80 \%$. Two cases had an open/elevated $\mathrm{MH}$, one case had an open/flat $\mathrm{MH}$ and one further case went on to have a total retinal detachment and reduced BCVA.

\section{MRS}

Fifty-three cases of MRS were identified from the retrospective study, which included 27 cases that underwent surgical management and 26 cases that were managed conservatively. Mean age in the surgical and conservative groups were 59.4 (range: 36-81) and 63.4 (range: 35-99) years, respectively (Table 2). Mean baseline BCVA was significantly different between the surgical group (0.87 $\log$ MAR; range: $0.3-1.9)$ and the conservative management group (0.45 $\log$ MAR; range: $-0.18-1.8) ; p=0.002)$.

All cases that had been managed surgically underwent PPV with 22/27 (81\%) having gas tamponade (Table 3) and two cases having peripheral laser retinopexy for peripheral retinal breaks combined with PPV. The ILM was peeled in $20 / 27(74.1 \%)$ cases and the most frequently used endotamponade was SF6 in 9/27 (33.3\%) cases. Mean BCVA remained stable in the surgically managed cases from a baseline of $0.87 \log$ MAR to 0.68 logMAR (range: $-0.2-3.0)$ post-operatively $(p=0.183)$. BCVA deteriorated in $5 / 27(18.5 \%)$ cases, it was unchanged in $3 / 27(11.1 \%)$ cases, and improved in 19/27 (70.3\%) cases. A total of 12 cases had an improvement in BCVA of greater than $0.3 \log$ Mar units or 2 Snellen lines (Fig. 2). The mean follow-up period was 11.3 months (range: 6 months to 5 years).

Anatomical outcomes showed a persistent MRS in 2/27 (7.4\%) cases, partially resolved MRS in 7/27 (25.9\%) cases and completely resolved MRS in 16/27 (59.2\%) cases (two cases undetermined). Of the cases with a co-existent macular hole, 4/5 (80\%) cases were successfully closed and one case remained open and flat.

\section{Discussion}

Our present study demonstrates that PPV is the preferred approach for the management of MHRD and MRS in the UK, bearing in mind that none of the cases reported had previous failed surgery. Primary macular hole closure rate was $25 \%$, and limited visual improvements were demonstrated in $60 \%$ of eyes. The MRS cases that were managed 
Table 2 Macular retinoschisis (MRS) cases: baseline characteristics

\begin{tabular}{|c|c|c|c|c|}
\hline & $\begin{array}{l}\text { Total } \\
n=52^{\mathrm{a}}\end{array}$ & $\begin{array}{l}\text { MRS surgical group } \\
\text { Mean }(\mathrm{CI}) n=27\end{array}$ & $\begin{array}{l}\text { MRS conservative group } \\
\text { Mean }(\mathrm{CI}) n=25^{\mathrm{a}}\end{array}$ & $p$-Value \\
\hline Mean age & 52 & $59.4(54.65-64.12)$ & $63.4(57.69-69.16)$ & 0.293 \\
\hline Mean baseline BCVA & 52 & $0.87(0.73-1.02)$ & $0.45(0.25-0.65)$ & 0.002 \\
\hline Gender & $n=53$ & $n(\%) n=27$ & $n(\%) n=26$ & \\
\hline Male & 20 & $12(30.8)$ & $8(44.4)$ & 0.305 \\
\hline Female & 33 & $15(69.2)$ & $18(55.6)$ & \\
\hline Axial length & $n=32^{\mathrm{b}}$ & $n(\%) n=22^{\mathrm{b}}$ & $n(\%) n=10^{\mathrm{b}}$ & \\
\hline $25.5-30.0 \mathrm{~mm}$ & 23 & $14(63.6)$ & $9(90.0)$ & 0.124 \\
\hline$>30.0 \mathrm{~mm}$ & 9 & $8(36.4)$ & $1(10.0)$ & \\
\hline Lens status & $n=52^{\mathrm{a}}$ & $n(\%) n=27$ & $n(\%) n=25^{\mathrm{a}}$ & \\
\hline Clear & 30 & $16(59.3)$ & $14(56.0)$ & 0.656 \\
\hline Cataract & 8 & $3(11.1)$ & $5(20.0)$ & \\
\hline Posterior chamber IOL & 14 & $8(29.6)$ & $6(24.0)$ & \\
\hline \multicolumn{5}{|l|}{ Macular pathology } \\
\hline Posterior staphyloma & $n=52^{\mathrm{a}}$ & $n(\%) n=27$ & $n(\%) n=25^{\mathrm{a}}$ & \\
\hline No & 16 & $7(25.9)$ & $9(36.0)$ & 0.432 \\
\hline Yes & 36 & $20(74.1)$ & $16(64.0)$ & \\
\hline Non-foveal macular hole & $n=52^{\mathrm{a}}$ & $n(\%) n=27$ & $n(\%) n=25^{\mathrm{a}}$ & \\
\hline No & 49 & $24(88.9)$ & $25(100.0)$ & 0.086 \\
\hline Yes & 3 & $3(11.0)$ & $0(0.0)$ & \\
\hline Post-vitreous detachment & $n=52^{\mathrm{a}}$ & $n(\%) n=27$ & $n(\%) n=25^{\mathrm{a}}$ & \\
\hline No & 30 & 15 (55.6) & $15(60.0)$ & 0.746 \\
\hline Yes & 22 & $12(44.4)$ & $10(40.0)$ & \\
\hline Chorioretinal atrophy & $n=52^{\mathrm{a}}$ & $n(\%) n=27$ & $n(\%) n=25^{\mathrm{a}}$ & \\
\hline No & 35 & $15(55.6)$ & $20(80.0)$ & 0.060 \\
\hline Yes & 17 & $12(44.4)$ & $5(20.0)$ & \\
\hline Epiretinal membrane & $n=52^{\mathrm{a}}$ & $n(\%) n=27$ & $n(\%) n=25^{\mathrm{a}}$ & \\
\hline No & 35 & $15(55.6)$ & $20(80.0)$ & 0.060 \\
\hline Yes & 17 & $12(44.5)$ & $5(20.0)$ & \\
\hline Choroidal neovascularisation & $n=52^{\mathrm{a}}$ & $n(\%) n=27$ & $n(\%) n=25^{\mathrm{a}}$ & \\
\hline No & 51 & $27(100.0)$ & $24(96.0)$ & 0.294 \\
\hline Yes & 1 & $0(0.0)$ & $1(4.0)$ & \\
\hline Macular hole & $n=53$ & $n(\%) n=27$ & $n(\%) n=26$ & \\
\hline No & 48 & $22(81.5)$ & $26(100.0)$ & 0.041 \\
\hline Yes & 5 & $5(18.5)$ & $0(0.0)$ & \\
\hline
\end{tabular}

${ }^{a}$ Data missing in one case

${ }^{\mathrm{b}}$ More than one case surgically demonstrated anatomical improvements in $85 \%$ of cases and limited visual improvements in $70 \%$ of cases. One outlier skewed the visual acuity data (Fig. 2).

According to the data collected from the current BOSU study, the estimated incidence of MHRD in the UK is 0.0156 per 100,000 population per year. A total of 19 cases were identified originally by the BOSU yellow card reporting system; however, nine cases were excluded on the basis of two being over reported, three were diagnosed incorrectly, one was lost to follow-up and three failed to respond to the questionnaires that were sent out. Our estimate of the incidence of MHRD is therefore based on 10 cases reported by nine vitreoretinal surgeons and probably represents an underestimation of the true UK incidence of MHRD.

Traditionally PPV, ERM/ILM peeling with or without gas tamponade have been the primary treatments for MHRD. All MHRD cases reported in this series are primary cases, thus final reattachment and closure rates are not available which would explain the $25 \%$ reported closure rates. ILM peeling in addition to peeling of pre-retinal structures for MHRD are generally considered important to reduce traction and create 
Table 3 Surgical techniques in the surgical management of macular retinoschisis (MRS) including types of endotamponade used and posturing regimes

\begin{tabular}{ll}
\hline $\begin{array}{l}\text { Surgical } \\
\text { procedures }\end{array}$ & $\begin{array}{l}n(\%) n \\
=27\end{array}$ \\
\hline PVD induced & $16(59.3)$ \\
ERM peel & $15(55.6)$ \\
ILM peel & $20(74.1)$ \\
Stain & $19(70.4)$ \\
Retinopexy & $2(7.4)$ \\
Explant & $0(0.0)$ \\
Endotamponade & \\
$\quad$ None & $4(14.8)$ \\
Air & $1(3.7)$ \\
SF6 & $9(33.3)$ \\
C2F6 & $7(25.9)$ \\
C3F8 & $6(22.2)$ \\
Silicone/heavy & $0(0.0)$ \\
oil & \\
Posturing regime & \\
None & \\
$<24$ h & $6(22.2)$ \\
1-7 days & $4(14.8)$ \\
$>7$ days & $15(55.6)$ \\
\hline
\end{tabular}

SF6 sulphur hexafluoride, C2F6 hexafluoroethane, $C 3 F 8$ perfluoropropane

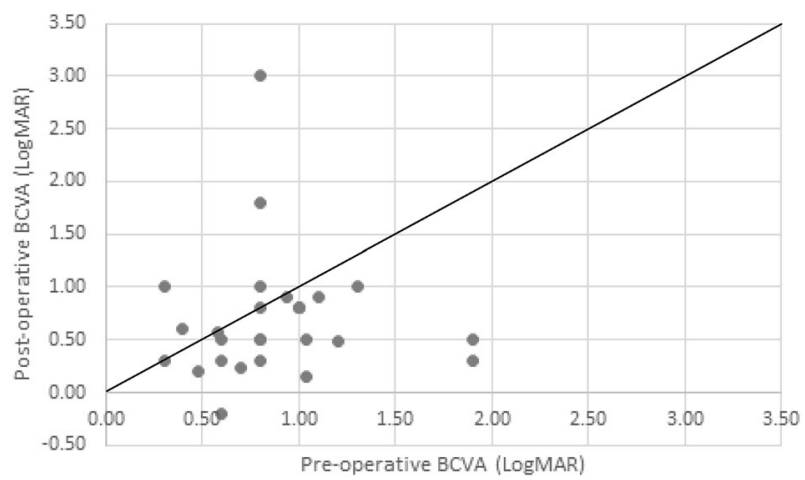

Fig. 2 BCVA (logMAR) change in the surgically managed eyes with MRS. The line of equality represents no change in BCVA, with the points below the line indicating an improvement in BCVA and those above the line indicating a reduction in post-operative BCVA. One outlier was noted as a result of a total post-operative retinal detachment managed by vitrectomy and oil and sustained a marked deterioration in the vision

greater retinal redundancy for the closure of the macular hole [29-31]. However, according to the literature available, macular hole closure rates using OCT monitoring have generally been low and varied from 10 to100\% and retinal reattachment rates have varied from 43 to $92 \%$ [29, 32-39]. Visual outcomes for MHRD have generally been disappointing when compared with other forms of surgery for vitreo-macular diseases. The large ranges for success indicate the complexity of these disorders and the variation in efficacy of the surgical techniques. Retinal reattachment is usually necessary to assess closure of the $\mathrm{MH}$, as a persistent large macular detachment is unlikely to coexist with a successfully closed MH, although persistent shallow SRF has been reported following surgery for idiopathic macular hole [32]. Therefore, closure rates for MH may be more appropriately quoted in the context of successfully reattached retinae. Clinically, an open but flat MH may be seen post-operatively, which has been attributed to inner retinal shortening and the lack of retinal redundancy which cannot be compensated for despite peeling of the ILM [40]. In our series, eyes with an open flat macular hole post-operatively had an improvement in visual acuity, but those with an open elevated macular hole post-operatively had worse visual acuity. Thus, retinal reattachment and a flat but open macular hole may be an acceptable outcome in some highly myopic eyes with posterior staphyloma.

Lim et al. reviewed 114 subjects with MHRD and reported better visual outcomes in eyes with retinal reattachment and macular hole closure, indicating the importance of the anatomical features based on OCT [32]. In keeping with our data, visual outcomes were dependent on macular hole closure although not a statistically significant finding in our report. Furthermore, greater age at presentation as well as the use of C3F8 tamponade were associated with a greater likelihood of anatomical success. We found older age to be associated with improved BCVA postoperatively and $\mathrm{C} 2 \mathrm{~F} 6$ to have a borderline association with improved BCVA post-operatively; however, these findings were not significant when carried through to multivariate analysis. Larger studies would be required to establish further associations. The presence of MRS in our study was significantly associated with failure of macular hole closure, in keeping with a review by Alkabes et al. who concluded that eyes with macular holes associated with foveoschisis required more procedures and had poorer visual outcomes compared with those without schisis [41].

The management of MRS depends on visual symptoms and OCT features as shown in the present study where eyes that underwent surgical intervention had worse baseline visual acuity compared to those managed conservatively. Thus, the timing of surgery for myopic MRS is driven clinically by the visual acuity at presentation. Whilst it is well documented that isolated MRS is rare and most eyes with myopic MRS remain stable, MRS may be coexistent with pre-retinal structures, epiretinal membrane, macular hole and macular detachment [7, 11, 19]. MRS may progress to macular hole and/or retinal detachment in some eyes and the presence of a foveal detachment is a well-documented risk factor for $\mathrm{MH}$ formation [19, 23, 42]. 
In eyes with myopic MRS, better visual outcomes have been associated with better pre-operative vision, shorter axial length and the reattachment of foveal photoreceptors in eyes with a pre-operative foveal detachment [43]. Ikuno et al. demonstrated BCVA improvement of more than 2 lines in three out of eight $(37.5 \%)$ eyes with $\mathrm{MH}$ associated with MRS following PPV with ILM peel and gas tamponade [44]. In eyes without a macular hole, visual acuity improvements of 2-5 lines and foveal reattachment have been demonstrated in $75-100 \%$ of patients following PPV and gas tamponade with or without ILM peel for MRS [23, 44-50]. Thus, PPV surgery with peeling of pre-retinal structures and gas tamponade with or without ILM peel for eyes with isolated myopic MRS is probably the procedure of choice, but visual outcomes are worse when retinal detachment or macular hole coexist [23].

PPV alone does not address the posterior staphyloma, which would suggest some benefit in posterior scleral reinforcement in the form of episcleral macular buckling (EMB) for patients with MHRD or MRS. Ando et al. in 2007 suggested that the preferred procedure for MHRD in highly myopic eyes with posterior staphyloma was EMB rather than PPV [51]. The authors report retinal reattachment rates of $93 \%$ and $100 \%$ after primary and secondary EMB surgery, respectively, compared to $50 \%$ after PPV and $86 \%$ after redo surgery with an EMB [51]. The visual outcomes were also significantly better in the EMB group compared to the PPV group [51]. Other retrospective case series of EMB using varying types of macular buckles have also demonstrated significant improvements in visual acuity and long-term maintenance of macular hole closure and retinal reattachment [25-29, 52-56]. In 2011, Devin et al. reported the successful use of a T-shaped scleral buckle which can be threaded along a 2-mm band that is subsequently secured nasal to the superior and inferior recti; this technique has the added advantages of avoidance of muscle disinsertion, no use of posterior sutures and the ability to visually adjust the height of the buckle [5, 28]. A more recent study by Mura et al. reported on 21 primary or secondary MHRD cases which either used the T-shaped MB alone or in conjunction with PPV and reported a final retinal reattachment rate of $100 \%$ and a $\mathrm{MH}$ closure rate of $90 \%$ [28]. EMB have also been used in the management of MRS with a number of studies recommending a combination of macular buckling and vitrectomy in order to address both inner traction and the posterior staphyloma [57-59]. Recent published data, however, using a variation on ILM peeling have demonstrated macular hole closure rates of $80-100 \%$ and retinal reattachment rates of $100 \%$ using the inverted ILM flap technique, compared to $36-55 \%$ and 55-91\%, respectively, using standard ILM peeling in cases with MHRD [60, 61].
In summary, macular buckling has not been widely adopted in the UK, and PPV surgery is generally considered the primary procedure of choice in eyes with MHRD and MRS where surgery is indicated. While it appears that a combined approach may yield better anatomic outcomes in MHRD cases, it remains unclear whether PPV alone would be a reasonable primary approach leaving EMB to those that fail primary surgery. The inverted ILM flap technique deserves further study as it may avoid the need for EMB. Limitations of the current study include the mixed study design and the small sample size. Larger numbers would be needed to demonstrate a significant change in visual outcomes given the limited scope for visual acuity improvements. Furthermore, the limited sample of vitreoretinal surgeons surveyed may not be representative of the whole UK practice. In order to represent current trends in the management of MRS and MHRD, it was necessary to involve different surgeons. However, inter-surgeon variation, and the variety of techniques used, may introduce additional confounding factors. Long-term visual acuity data of MRS cases that were managed conservatively are lacking, thus direct comparison with the surgically managed MRS group and identifying the optimal time for surgery were not possible.

In conclusion, we present a study of primary PPV for the management of MHRD and MRS. Macular hole primary closure rates are low, which would suggest the need to consider EMB either as a primary or secondary procedure, but variations in surgical technique such as the use of the inverted ILM flap technique deserve further study. Anatomic and visual outcomes of the management of MRS are similar to those reported in the literature.

\section{Summary}

\section{What was known before}

- Pathological myopia (PM) is a common cause of visual loss worldwide.

- Among the spectrum of complications associated with PM, macular retinoschisis (MRS) and macular hole retinal detachment (MHRD) have been notoriously difficult to manage due to axial elongation of the globe, posterior staphyloma and chorioretinal atrophy.

- Pars plana vitrectomy (PPV), encircling scleral buckle, peeling of ILM/pre-retinal membranes, various intraocular tamponade agents and macular buckle have all been used with variable success rates.

- Traditionally, management in the UK has involved PPV, epiretinal membrane and internal limiting membrane peel with gas or oil tamponade but the literature regarding outcomes is limited.

- According to the international literature available, macular hole closure rates using OCT monitoring have 
generally been low and varied from 10 to $100 \%$ and retinal reattachment rates have varied from 43 to $92 \%$.

- The surgical management of MRS has generally demonstrated significant anatomical and visual improvements.

\section{What this study adds}

- Our present study demonstrates that PPV is the preferred approach for the primary surgical management of MHRD and MRS in the UK.

- Primary macular hole closure rate was $25 \%$, and limited visual improvements were demonstrated in $60 \%$ of eyes.

- Anatomic and visual improvements were noted in $85 \%$ and $70 \%$, respectively, of MRS cases managed surgically.

Acknowledgements The British Ophthalmological Surveillance Unit, The Royal College of Ophthalmologists, The COBRA Study Group and The Whipps Cross University Hospital Charitable Trust.

COllaboration of British RetinAl Surgeons (COBRA) Stephen J. Charles, David Charteris, Richard J. Haynes, D. Alistair H. Laidlaw, David H. Steel, David Yorston, Tom H. Williamson, Hadi Zambarakji.

\section{Compliance with ethical standards}

Conflict of interest The authors declare that they have no conflict of interest.

\section{References}

1. Wong T, Ferreira A, Hughes R, Carter G, Mitchell P. Epidemiology and disease burden of pathologic myopia and myopic choroidal neovascularization: an evidence-based systematic review. Am J Ophthalmol. 2014;157:9-25.

2. The Eye Diseases Prevalence Research Group. The prevalence of refractive errors among adults in the United States, Western Europe, and Australia. Arch Ophthalmol. 2004;122:495-505.

3. Grossniklaus H, Green W. Pathologic findings in pathologic myopia. Retina. 1992;12:127-33.

4. Ohno-Matsui K, Kawasaki R, Jonas J, Cheung C, Saw S, Verhoeven $\mathrm{V}$, et al. International photographic classification and grading system for myopic maculopathy. Am J Ophthalmol. 2015;159:877-83.e7.

5. Devin F, Tsui I, Morin B, Duprat J, Hubschman J. T-shaped scleral buckle for macular detachments in high myopes. Retina. 2011;31:177-80.

6. Ortisi E, Avitabile T, Bonfiglio V. Surgical management of retinal detachment because of macular hole in highly myopic eyes. Retina. 2012;32:1704-18.

7. Baba T, Ohno-Matsui K, Futagami S, Yoshida T, Yasuzumi K, Kojima A, et al. Prevalence and characteristics of foveal retinal detachment without macular hole in high myopia. Am J Ophthalmol. 2003;135:338-42.

8. Hsiang H, Ohno-Matsui K, Shimada N, Hayashi K, Moriyama M, Yoshida T, et al. Clinical characteristics of posterior staphyloma in eyes with pathologic myopia. Am J Ophthalmol. 2008;146: 102-10.
9. Wu P, Chen Y, Chen Y, Chen C, Shin S, Tsai C, et al. Factors associated with foveoschisis and foveal detachment without macular hole in high myopia. Eye (Lond). 2009;23:356-61.

10. Akiba J, Konno S, Yoshida A. Retinal detachment associated with a macular hole in severely myopic eyes. Am J Ophthalmol. 1999;128:654-5.

11. Takano M, Kishi S. Foveal retinoschisis and retinal detachment in severely myopic eyes with posterior staphyloma. Am J Ophthalmol. 1999;128:472-6.

12. Baba T, Tanaka S, Maesawa A, Teramatsu T, Noda Y, Yamamoto S. Scleral buckling with macular plombe for eyes with myopic macular retinoschisis and retinal detachment without macular hole. Am J Ophthalmol. 2006;142:483-7.

13. Zhu. Z, Ji X, Zhang J, Ke G. Posterior scleral reinforcement in the treatment of macular retinoschisis in highly myopic patients. Clin Exp Ophthalmol. 2009;37:660-3.

14. Shimada N, Tanaka Y, Tokoro T, Ohno-Matsui K. Natural course of myopic traction maculopathy and factors associated with progression or resolution. Am J Ophthalmol. 2013;156:948-57.e1.

15. Sun. C, Liu Z, Xue. A, Yao K. Natural evolution from macular retinoschisis to full-thickness macular hole in highly myopic eyes. Eye (Lond). 2010;24:1787-91.

16. Shao Q, Xia H, Heussen F, Ouyang Y, Sun X, Fan Y, et al. Postoperative anatomical and functional outcomes of different stages of high myopia macular hole. BMC Ophthalmol. 2015;15:93.

17. Panozzo G, Mercanti A. Vitrectomy for myopic traction maculopathy. Arch Ophthalmol. 2007;125:767-72.

18. Smiddy W, Kim S, Lujan B, Gregori G. Myopic traction maculopathy: spectral domain optical coherence tomographic imaging and a hypothesized mechanism. Ophthalmic Surg Lasers Imaging. 2009;40:169-73.

19. Gaucher D, Haouchine B, Tadayoni R, Massin P, Erginay A. Long-term follow-up of high myopic foveoschisis: natural course and surgical outcome. Am J Ophthalmol. 2007;143:455-62.

20. Ikuno Y, Tano Y. Early macular holes with retinoschisis in highly myopic eyes. Am J Ophthalmol. 2003;136:741-800.

21. Shimada N, Ohno-Matsui K, Baba T, Futagami S, Tokoro T. Natural course of macular retinoschisis in highly myopic eyes without macular hole or retinal detachment. Am J Ophthalmol. 2006;142:497-500.

22. Matsumura N, Ikuno Y, Tano Y. Posterior vitreous detachment and macular hole formation in myopic foveoschisis. Am J Ophthalmol. 2004;138:1071-3.

23. Gohil R, Sivaprasad S, Han L, Mathew R, Kiousis G, Yang Y. Myopic foveoschisis: a clinical review. Eye. 2015;29:593-601.

24. Laviers H, Zambarakji $\mathrm{H}$. Management of macular hole retinal detachment and macular retinoschisis secondary to pathological myopia: a national survey of UK practice patterns. Eye. 2013;27: 1324.

25. Ripandelli G, Coppe A, Fedeli R, Parisi V, D'Amico D, Stirpe M. Evaluation of primary surgical procedures for retinal detachment with macular hole in highly myopic eyes: A randomised comparison of vitrectomy versus posterior episcleral buckling surgery. Ophthalmology. 2001;108:2258-64.

26. Theodossiadis G, Theodossiadis P. The macular buckling procedure in the treatment of retinal detachment in highly myopic eyes with macular hole and posterior staphyloma: mean follow-up of 15 years. Retina. 2005;25:285-9.

27. Siam A, El Maamoun T, Ali M. Macular buckling for myopic macular hole retinal detachment: a new approach. Retina. 2012;32:748-53.

28. Mura M, Iannetta D, Buschini E, de Smet M. T-shaped macular buckling combined with $25 \mathrm{G}$ pars plana vitrectomy for macular hole, macular schisis, and macular detachment in highly myopic 
eyes. Br J Ophthalmol. 2016;101:383-8. https://doi.org/10.1136/ bjophthalmol-2015-308124.

29. Uemoto R, Yamamoto S, Tsukahara I, Takeuchi S. Efficacy of internal limiting membrane removal for retinal detachments resulting from a myopic macular hole. Retina. 2004;24:560-6.

30. Su J, Liu Z, Zheng L, Cui H. Vitrectomy with internal limiting mebrane peel vs no-peeling for macular hole induced retinal detachment (MHRD); a meta-analysis. BMC Ophthalmol. 2015; 15:62.

31. Gao X, Guo J, Meng X, Wang J, Peng X, Ikuno Y. A metaanalysis of vitrectomy with or without internal limiting membrane peeling for macular hole retinal detachment in the highly myopic eyes. BMC Ophthalmol. 2016;16:87.

32. Lim L, Tsai A, Wong D, Wong E, Yeo I, Loh B, et al. Prognostic factor analysis of vitrectomy for retinal detachment associated with myopic macular holes. Ophthalmology. 2014;121:305-10.

33. Ichibe M, Yoshizawa T, Murakami K, Ohta M, Oya Y, Yamamoto S, et al. Surgical management of retinal detachment associated with myopic macular hole: anatomic and functional status of the macula. Am J Ophthalmol. 2003;136:277-84.

34. Ikuno Y, Sayanagi K, Oshima T, Gomi F, Kusaka S, Kamei M, et al. Optical coherence tomographic findings of macular holes and retinal detachment after vitrectomy in highly myopic eyes. Am J Ophthalmol. 2003;136:477-81.

35. Kadonosono K, Yazama F, Itoh N, Uchio E, Nakamura S, Akura $\mathrm{J}$, et al. Treatment of retinal detachment resulting from myopic macular hole with internal limiting membrane removal. Am J Ophthalmol. 2001;131:203-7.

36. Chen. Y, Chen. T, Yang K, Lee W, Kuo Y, Chao A, et al. Treatment of retinal detachment resulting from posterior staphyloma-associated macular hole in highly myopic eyes. Retina. 2006;26:25-31.

37. Mitry D, Zambarakji H. Recent trends in the management of maculopathy secondary to pathological myopia. Graefes Arch Clin Exp Ophthalmol. 2012;250:3-13.

38. Feng L, Jin X, Li J, Zhai J, Fang W, Mo J, et al. Surgical management of retinal detachment resulting from macular hole in a setting of high myopia. Eye Sci. 2012;27:69-75.

39. Meng L, Wei W, Li Y, Han X, Shi X, Yang M. Treatment of retinal detachment secondary to macular hole in highly myopic eyes: pars plana vitrectomy with internal limiting membrane peel and silicone oil tamponade. Retina. 2014;34:470-6.

40. Gentile R, Landa G, Pons M, Eliott D, Rosen R. Macular hole formation, progression, and surgical repair: case series of serial optical coherence tomography and time lapse morphing video study. BMC Ophthalmol. 2010;10:24.

41. Alkabes M, Pichi F, Nucci P, Massaro D, Dutra Medeiros M, Corcisteugi B, et al. Anatomical and visual outcomes in high myopic macular hole (HM-MH) without retinal detachment: a review. Graefes Arch Clin Exp Ophthalmol. 2014;252:191-9.

42. Burés-Jelstrup A, Alkabes M, Gómez-Resa M, Rios J, Corcóstegui B, Mateo C. Visual and anatomical outcome after macular buckling for macular hole with associated foveoschisis in highly myopic eyes. Br J Ophthalmol. 2014;98:104-9.

43. Kumagai K, Furukawa M, Ogino N, Larson E. Factors correlated with postoperative visual acuity after vitrectomy and internal limiting membrane peeling for myopic foveoschisis. Retina. 2010;30:874-80.

44. Ikuno Y, Tano Y. Vitrectomy for macular holes associated with myopic foveoschisis. Am J Ophthalmol. 2006;141:774-6.
45. Ikuno Y, Sayanagi K, Ohji M, Kamei M, Gomi F, Harino S, et al. Vitrectomy and internal limiting membrane peeling for myopic foveoschisis. Am J Ophthalmol. 2004;137:719-24.

46. Ikuno Y, Sayanagi K, Soga K, Oshima Y, Ohji M, Tano Y. Foveal anatomical status and surgical results in vitrectomy for myopic foveoschisis. Jpn J Ophthalmol. 2008;52:269-76.

47. Fang X, Weng Y, Xu S, Chen Z, Liu J, Chen B, et al. Optical coherence tomographic characteristics and surgical outcome of eyes with myopic foveoschisis. Eye. 2009;23:1336-42.

48. Kwok A, Lai T, Yip W. Vitrectomy and gas tamponade without internal limiting membrane peeling for myopic foveoschisis. Br J Ophthalmol. 2005;89:1180-3.

49. Sayanagi K, Ikuno Y, Tano Y. Reoperation for persistent myopic foveoschisis after primary vitrectomy. Am J Ophthalmol. 2006;141:414-7.

50. Furukawa M, Ogino N, Larson E. Factors correlated with postoperative visual acuity after vitrectomy and internal limiting membrane peeling for myopic foveoschisis. Retina. 2010;30: 874-80.

51. Ando F, Ohba N, Touura K, Hirose H. Anatomical and visual outcomes after episcleral macular buckling compared with those after pars plana vitrectomy for retinal detachment caused by macular hole in highly myopic eyes. Retina. 2007;27:37-44.

52. Tanaka T, Ando F, Usui M. Episcleral macular buckling by semirigid shaped-rod exoplant for recurrent retinal detachment with macular hole in highly myopic eyes. Retina. 2005;25:147-51.

53. Sasoh M, Yoshida S, Ito Y, Matsui K, Osawa S, Uji Y. Macular buckling for retinal detachment due to macular hole in highly myopic eyes with posterior staphyloma. Retina. 2000;20: 445-9.

54. Tian J, Tang L, Guo X, Luo Y. Episcleral macular buckling for posterior retinal detachment in silicone oil filled eyes associated with myopic macular hole. Int J Ophthalmol. 2013;6:165-8.

55. Forlini M, Szkaradek M, Rejdak R, Bratu A, Rossini P, D’Eliseo D, et al. Modification of adjustable macular buckling with $29-\mathrm{G}$ chandeleier light for optimal positioning in highly myopic eyes with macular hole. Retin Cases Brief Rep. 2016;11:249-54.

56. Alkabes M, Burés-Jelstrup A, Salinas C, Medeiros M, Rios J, Corcostegui B, et al. Macular buckling for previously untreated and recurrent retinal detachment due to high myopic macular hole: a 12-month comparative study. Graefes Arch Clin Exp Ophthalmol. 2014;252:571-81.

57. Qi Y, Duan A, You Q, Jonas J, Wang N. Posterior scleral reinforcement and vitrectomy for myopic foveoschisis in extreme myopia. Retina. 2015;35:351-7.

58. Parolini B, Frisina R, Pinackatt S, Gasparotti R, Gatti E, Baldi A, et al. Indications and results of a new L-shaped macular buckle to support a posterior staphyloma in high myopia. Retina. 2015;35: 2469-82.

59. Mateo C, Jelstrup AB, Navarro R, Corcostegui B. Macular buckling for eyes with myopic foveoschisis secondary to posterior staphyloma. Retina. 2012;32:1121-8.

60. Sasaki H, Shiono A, Kogo J, Yomoda R, Munemasa Y, Syoda M, et al. Inverted internal limiting membrane flap technique as a useful procedure for macular hole-associated retinal detachment in highly myopic eyes. Eye (Lond). 2017;31:545-50.

61. Baba R, Wakabayashi Y, Umazume K, Ishikawa T, Yagi H, Muramatsu D, et al. Efficacy of the inverted internal limiting membrane flap technique with vitrectomy for retinal detachment associated with myopic macular holes. Retina. 2017;37:466-71. 